\title{
Hereditary motor end-plate disease in the mouse: light and electron microscopic studies
}

\author{
L. W. DUCHEN \\ From the Department of Neuropathology, Institute of Psychiatry and The Maudsley Hospital, \\ London S.E.5
}

With a genetic appendix by A. G. Searle

From the M.R.C. Radiobiology Unit, Harwell, Berkshire

\begin{abstract}
Hereditary neuromuscular disorders in mice provide useful models for the study of the pathogenesis of many different disease processes and the reactions which they evoke in the nervous system. The clinical and light microscopic features of a fatal hereditary disease characterized by progressive muscular weakness were briefly described by Searle (1962) and by Duchen, Searle, and Strich (1967). The pathological changes which were found suggested that the primary abnormality in this disease was located in the region of the motor nerve terminals, and the name 'motor end-plate disease' seemed appropriate. This paper presents the results of recent light and electron microscopic studies of the disease which is now known to be due to an abnormality in the function of motor nerve terminals.
\end{abstract}

\section{THE CLINICAL SYNDROME}

This hereditary disease is transmitted by an autosomal recessive gene (med). The heterozygous carriers of the gene are clinically normal and no abnormalities are seen on histological examination of their neuromuscular system. About $25 \%$ of the offspring of matings of mice heterozygous for the gene are expected to be homozygous (med/med, henceforth called med mice) and show signs of the disease (see genetic appendix by Searle). At birth and for the next eight to 10 days homozygous med mice cannot be distinguished from their norma littermates. The first signs of abnormality are un steadiness of gait, the mouse swaying slightly from side to side, with a nodding movement of the head. Within the next two to three days muscular weaknes\& becomes increasingly severe and by the 12 th to $14 \mathrm{th}$ day of age muscle wasting is apparent. The mouse drags the hind legs behind the trunk (Fig. 1a), the forelimbs cannot be extended and cannot properly support the body so that the animal tends to lie in a prone position with the head held low. Righting reflexes are present but the mouse tends to fall over because of muscular weakness and takes longer

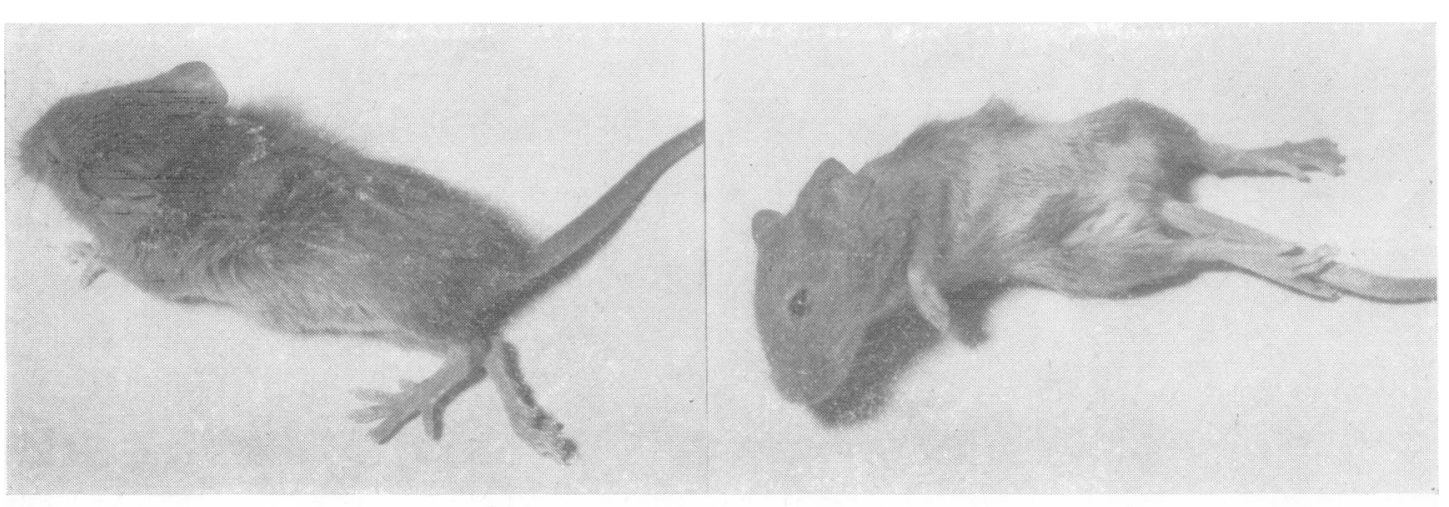

(a) (b)

FIG. 1. An 18-day-old 'med' mouse. (a) The hindlimbs are dragging because of weakness of the proximal thigh muscles. (b) The mouse has fallen over because of muscular weakness and can right itself though with difficulty. 
than normal to right itself (Fig. 1b). The progressive weakness affects especially the proximal muscles of all limbs. The thigh can scarcely be flexed to the trunk. By the 18 th to 19 th day the weakness is so severe that the mouse cannot make effective movements. Most affected animals die at 19 to 23 days of age. Occasionally they have survived until the 26th to 29 th day, but some survive for only 15 days. The cause of death is not entirely understood but clearly the homozygotes have difficulty in obtaining sufficient milk, since they have to compete in suckling with their unaffected littermates and cannot eat solid food or drink from the water-bottle available in the cage. Sensory systems are not apparently affected. Administration of anticholinesterase agents was attempted in a few affected mice but did not produce any significant effect on the muscular weakness.

\section{MATERIAL AND METHODS}

The studies described in this paper are the result of histological, histochemical, and electron microscopic examination of more than 70 affected (med/med) mice. Some mice were killed at $\mathbf{1 0}$ days of age when there was early but definite clinical evidence of the disease. The longest surviving homozygous mouse examined was 29 days old. For preparation of tissues for light microscopy the animals were killed with chloroform or with ether and, after removing the skin and opening the body cavities, were fixed entire in formol-calcium $(10 \%$ formalin in $1 \%$ calcium acetate) or formol-alcohol $(10 \%$ formalin in $60 \%$ alcohol). After decalcification in formiccitrate $(90 \%$ formic acid- 35 parts; $20 \%$ sodium citrate65 parts) blocks were taken of all limbs, the entire trunk including spinal cord, and head including the brain and brain-stem. Blocks of limbs were embedded in paraffin wax and sectioned in longitudinal and transverse planes and much use was made of the 'serial block' technique of Beesley and Daniel (1956) in order to obtain transverse sections at many levels of limbs, spinal cord, and brain. Paraffin sections were stained by various routine histological methods including cresyl violet, with and without luxol fast blue; Palmgren's silver method; periodic acidSchiff; and Sudan black. Silver impregnation of nerve fibres by a modification of Schofield's method (1960) was carried out on serial frozen sections cut at $60 \mu$ from blocks of limbs, trunks, and entire heads embedded in gelatin. Cholinesterase was demonstrated in serial $20 \mu$ longitudinal frozen sections of entire limbs fixed by perfusion with cold $\left(4^{\circ} \mathrm{C}\right)$ formol-calcium. The method used was essentially that of Koelle and Friedenwald (1949) modified by Lewis (1961) and Henderson (1967). The substrates used were acetyl- or butyrylthiocholine iodide at a $p \mathrm{H}$ of either $5 \cdot 2$ or $5 \cdot 6$, with and without prior incubation for 30 minutes in tetra-isopropylpyrophosphoramide (iso-OMPA) in a concentration of $3 \times 10^{-6} \mathrm{M}$. The method of Namba, Nakamura, and Grob (1967) was used for silver impregnation of nerve fibres in frozen sections after the completion of the cholinesterase reaction.

The distribution in the muscles of the forelimb of two enzymes, succinate dehydrogenase and phosphorylase, was studied in transverse cryostat sections of fresh tissue frozen in iso-pentane cooled with liquid nitrogen. Succinate dehydrogenase was demonstrated by the method of Nachlas et al. using Nitro-BT as described by Pearse (1960). Phosphorylase was demonstrated by the method of Lake (unpublished).

Electron microscopic studies were confined to $\mathrm{m}$. biceps brachii after light microscopy had shown this muscle to be severely abnormal in all med mice. Under ether anaesthesia the skin over m. biceps brachii was reflected laterally and the muscle covered by a pool of cold $\left(4^{\circ} \mathrm{C}\right)$ glutaraldehyde $(2.5 \%$ in phosphate buffer $p H$ 7.3). When the muscle was firm and yellow the biceps was dissected out, pinned on cork board, and fixed for one to two hours in fresh cold glutaraldehyde. In more recent work the animal was perfused through the left ventricle with cold buffered glutaraldehyde before the biceps was dissected out and treated as described before. Blocks were taken from the region of motor innervation of biceps, washed overnight in buffer, post-fixed in $1 \%$ osmium tetroxide, and dehydrated in graded alcohols. Blocks were stained in saturated alcoholic uranyl acetate and embedded in epoxy resin. In some cases blocks were washed in cold buffer after fixation and then immersed in the substrate (acetylthiocholine iodide-copper sulphateglycine) solution at $4^{\circ} \mathrm{C}$ and $p \mathrm{H} 5.2$ for five to 15 minutes for the demonstration of cholinesterase. No inhibitors of pseudocholinesterase were used. The blocks were then washed and placed for a few minutes in cold $1 \%$ sodium sulphide solution in dilute acetic acid, washed overnight in cold buffer, post-fixed in osmium tetroxide, dehydrated, and embedded. Sections were stained with lead citrate. Tissues from many normal control animals, usually littermates of med mice but showing no clinical evidence of disease, were treated for both light and electron microscopy in the same way as those from homozygous mice.

\section{LIGHT MICROSCOPIC OBSERVATIONS}

The characteristic histological abnormalities seen in this disease were atrophy of skeletal muscle fibres and a remarkable sprouting of the motor nerve fibres innervating them. No changes were found which suggested a primary myopathic disease. No morphological abnormalities were found in the brain or brain-stem, in the ventral horn cells of the spinal cord, or in peripheral nerves proximal to the motor end-plates.

In the affected med mice aged 10 to 15 days there were usually only mild abnormalities in the histological appearance of skeletal muscles. In sections of paraffin-embedded tissues the diameter of muscle fibres was sometimes less than in normal littermates. At this stage the subneural apparatuses did not appear abnormal and no ultraterminal nerve fibres were present. As the disease progressed it was found 


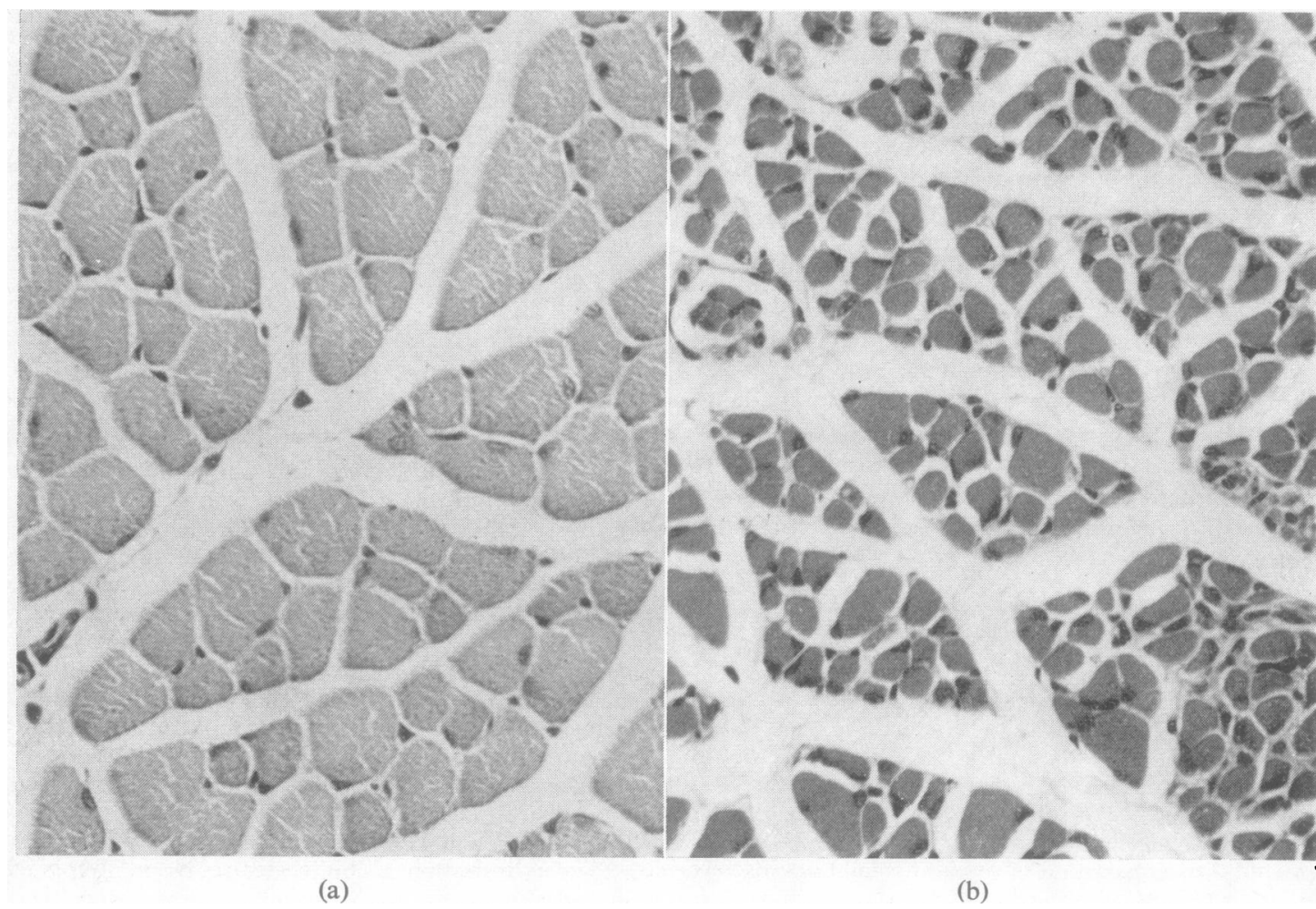

FIG. 2. Transverse section of the short head of m. biceps brachii of a normal 22-day-old mouse (a) and of a 'med' mous aged 24 days (b). There is severe atrophy of almost all fibres in the affected muscle. $H$ and $E, \times 400$.

that some muscles showed very much more marked abnormalities than others. The distribution of the severely affected muscles was symmetrical and was the same in all the mice studied. The short head of $\mathrm{m}$. biceps brachii was uniformly very abnormal histologically as were some other muscles of the shoulder girdle and also $\mathrm{m}$. rectus femoris. Muscles which were less severely and less uniformly abnormal histologically were the long head of the biceps brachii, triceps brachii, tibialis anterior, and gastrocnemius muscles. In other muscles fewer fibres were affected but it seemed that no muscle was entirely normal. In older med mice most muscle fibres of m. biceps brachii were severely atrophied (Fig. 2) while in other muscles, such as the triceps (Fig. 3) atrophied fibres were found scattered throughout the muscle among fibres of larger diameter. The atrophied fibres lay singly or in small groups. There was no fascicular arrangement of atrophied fibres. With longer survival the degree of atrophy and the proportion of muscle fibres affected seemed to become greater. The nuclei of the atrophied muscle fibres were usually situated subjacent to the sarco- lemma. No chains of nuclei were seen in longitudinal sections and no necrotic muscle fibres were found.

Silver impregnation of thick frozen sections showed that there was marked sprouting of motor nerve fibres at their terminals. Instead of the normal fairly simple terminal arborization (Fig. 4a) the motor nerve terminals were enlarged and complex and branching nerve fibres spread away from the region of the motor end-plate along the muscle fibre and also across adjacent muscle fibres. These sprouting nerve fibres were of very irregular diameter, some fibres had a beaded appearance and little knobs were present at their tips. The preterminal motor nerve fibres did not appear to be branched. No fragmentation or degeneration of preterminal axons was seen. In the mice surviving 19 days or more the appearance of the innervation of the most severely affected muscles was very abnormal. The atrophy of muscle fibres resulted in marked crowding together of nerve fibres and this, together with the profuse nerve sprouting, caused the muscle to appear to contain motor nerves greatly in excess of normal (Fig. 4b). 


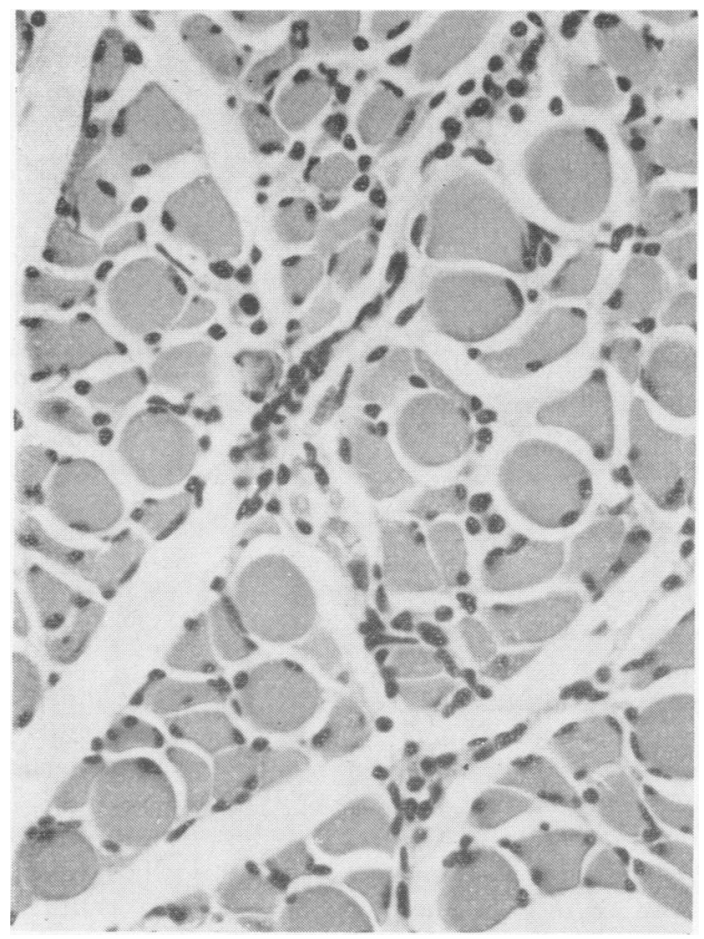

FIG. 3. M. triceps brachii of a 22-day-old 'med' mouse shows many atrophied muscle fibres scattered among fibres of more normal diameter. There is no fascicular distribution of atrophied fibres. $H$ and $E, \times 400$.
Marked abnormalities developed in the appearance of subneural apparatuses in sections stained to demonstrate cholinesterase (Fig. 5). The most severe abnormalities in cholinesterase preparations were seen in those muscles with the most severe degree of atrophy and the most abundant motor nerve sprouting. Instead of the normal regular complex of gutters (Fig. 6a) the subneural apparatuses were elongated, irregular in size and shape, and had elongated branching projections. It seemed that the distribution of cholinesterase followed the sprouting motor nerve fibres, and this was particularly marked in the enzyme preparations incubated for 20 minutes in substrate (either acetylor butyrylthiocholine) at $p \mathrm{H} 5.6$. The enzyme activity along the newly formed motor nerve sprouts

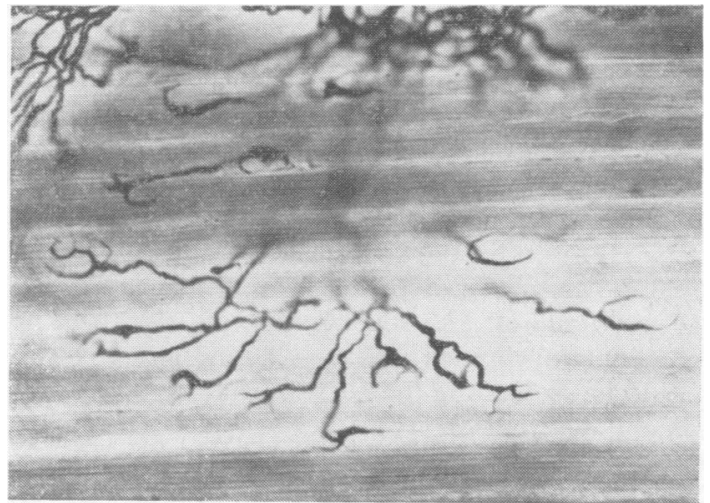

FIG. $4 a$

FIG. $4 b$

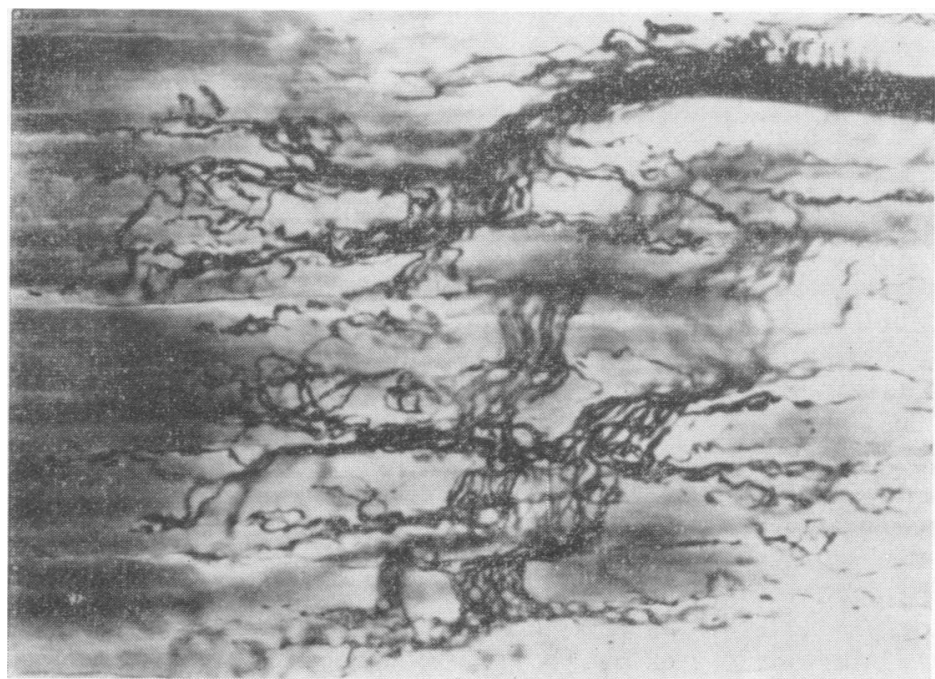

FIG. 4. Silver impregnation of m. biceps brachii of a normal mouse (a) and its 'med' littermate aged 19 days (b). In the normal muscle each motor nerve fibre ends in a simple arborization. In the 'med' muscle the nerve fibres are crowded together and there is sprouting from almost all nerve terminals so that the muscle contains more nerve fibres than normal. Schofield, $\times 300$. 


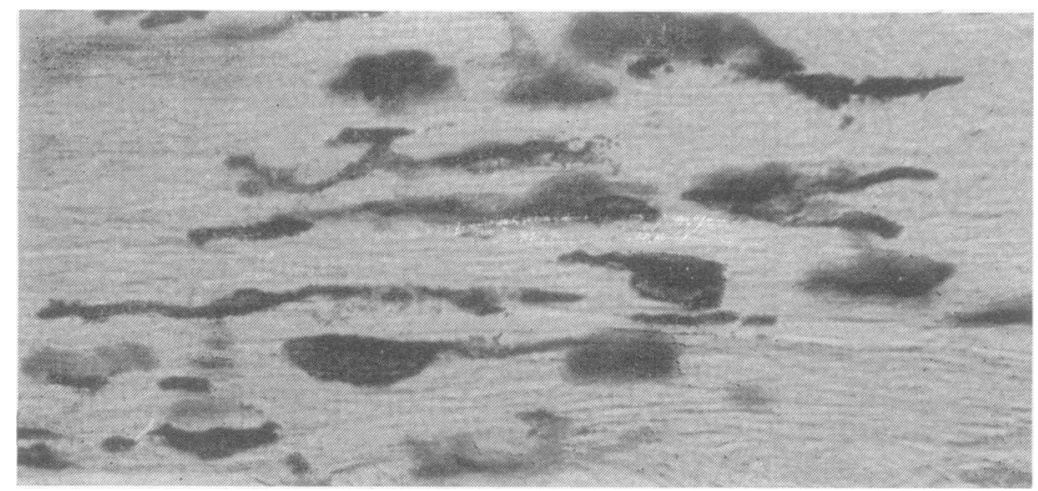

FIG. 5. Cholinesterase localization in $m$. biceps brachii of a 'med' mouse aged 19 days. The reaction product is seen in long branching lines extending from the gutters of the end-plates (compare with Fig. 6). There is no reaction product along preterminal axons. Acetylthiocholine substrate, $\mathrm{pH} \mathrm{5.6,}$ incubated 20 minutes. $\times 400$.

was largely abolished by pre-incubation for 30 minutes in iso-OMPA. Normally both true (acetyl) cholinesterase and non-specific cholinesterase are present at the end-plate, the reaction for the former being much the stronger. In the muscles of the med mice it seemed that most of the enzyme associated with the motor nerve sprouts was non-specific cholinesterase. The sections stained to demonstrate nerve fibres after the cholinesterase reaction showed that the motor nerve sprouts arose from the terminals (Fig. 6b and c). The sprouts did not arise from preterminal myelinated axons. Cholinesterase activity could be seen along the nerve sprouts in an irregular patchy distribution.

DISTRIBUTION OF SUCCINATE DEHYDROGENASE AND PHOSPHORYLASE The distribution of the enzymes succinate dehydrogenase and phosphorylase in muscle fibres of the forelimb was studied in 22 med mice aged 15 to 29 days and six normal mice (littermates of med) aged 15 to 19 days. In the muscles of the normal 15-day animal there was already differentiation of muscle fibres into two main classes, and this differentiation was more clearly seen with increasing age and the intensity of the staining became stronger. By the 19th day the histochemical differentiation of the muscle fibres and the distribution of the two main types was very like that of the adult mouse. In the superficial parts of $\mathrm{m}$. biceps and triceps brachii most of the muscle fibres gave a strongly positive reaction for phosphorylase, while the fibres rich in succinate dehydrogenase activity were few in number (Fig. 7). In the deep parts of the muscles the majority of fibres appeared to be rich in succinate dehydrogenase and fewer fibres gave a strong phosphorylase reaction. There were consistent changes in muscles of all the med mice studied histochemically (Fig. 8) biceps being affected more severely and uniformly than triceps, but in both these muscles the changes were progressive with longer survival. Succinate dehydrogenase activity was demonstrable in some muscle fibres even in severely atrophied fibres of the biceps in the longest surviving animal. The sequence of changes in the phosphorylase reaction was very different from that of succinate dehydrogenase. In 16-day-old med mice there was a great reduction in the intensity of the colour reaction for phosphorylase in many of the muscle fibres of both biceps and triceps muscles. By the 19th day very few of the fibres in the shoft head of biceps, where the atrophy was uniformp severe, showed any colour reaction and even these were weaker than normal and by the 23 rd and 298 days the phosphorylase reaction was almost absen. In triceps the abnormalities were always less severe than in biceps of the same animal and there were usually some fibres which were not apparently atrophied and which gave a stronger phosphorylase reaction than the other smaller atrophied fibres. In the longest surviving mouse the colour reaction for phosphorylase in triceps was weaker than in $\mathbb{D}$ earlier stages of the disease. It seemed clear that the atrophy of the muscle fibres and the weakening of the phosphorylase reaction were both progressive.

MUSCLE SPINDLES AND OTHER SENSORY STRUCTURES Intrafusal muscle fibres of normal size and with a strong phosphorylase colour reaction were often seen among weakly reacting extrafusal fibres. Although the state of the motor innervation of the intrafusal fibres was not determined with certainty, the annulospiral sensory nerve endings appeared normal. The innervation of other sensory structures such as Pacinian corpuscles and vibrissae and of skin and mucosae appeared normal.

\section{ELECTRON MICROSCOPIC OBSERVATIONS}

Electron microscopic examination of $\mathrm{m}$. biceps brachii from normal mice showed the characteristic $\mathrm{C}$ well-known appearance of motor nerve terminals 


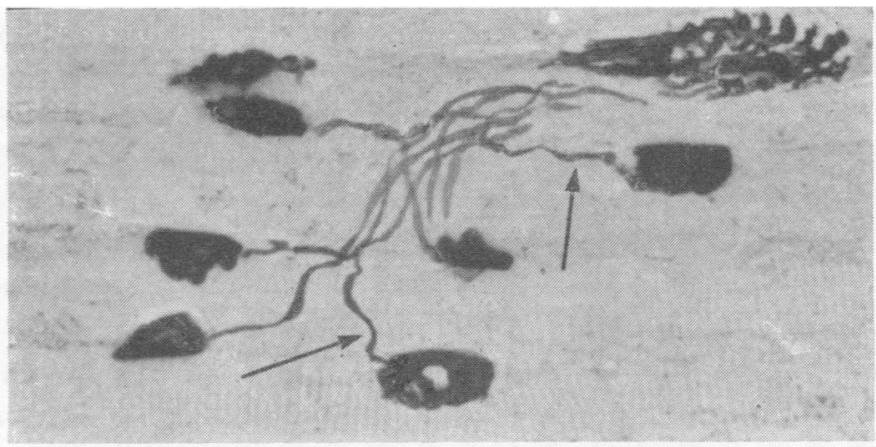

FIG. 6a

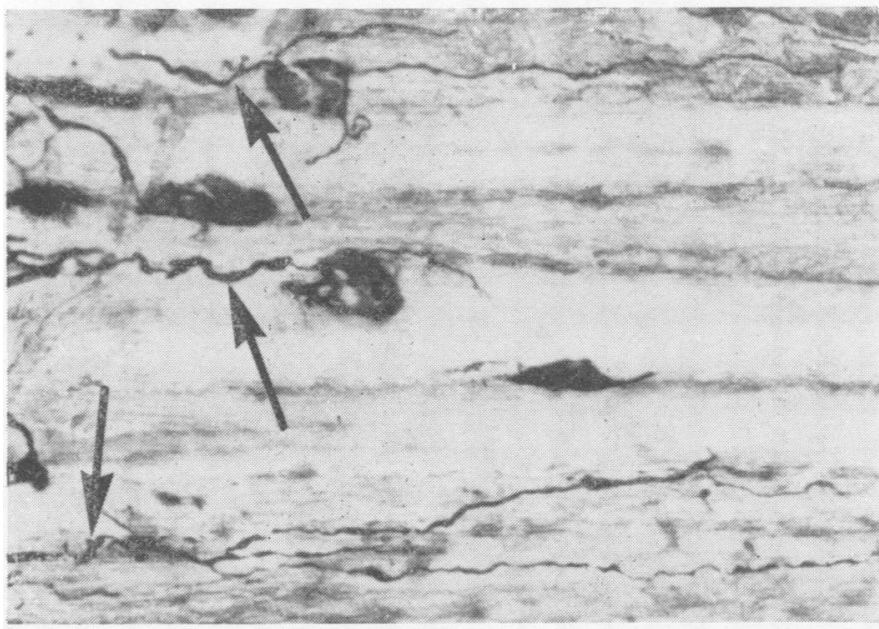

FIG. 6. Combined cholinesterase and silver impregnation of $m$. biceps brachii of normal (a) and 'med' mice (b and c) 26 days old. Each preterminal axon (arrow's to some) innervates one motor end-plate. Cholinesterase is localized to the subneural apparatuses in the normal and there are no ultraterminal nerve fibres. In the 'med' muscle nerve sprouts extend beyond the end-plates and show patchy cholinesterase activity (compare with Fig. 5). Acetylthiocholine substrate, $\mathrm{pH} 5 \cdot 2$, incubated 15 minutes. Namba et al., a and b $\times 400$; c $\times 600$.

FIG. $6 \mathrm{~b}$

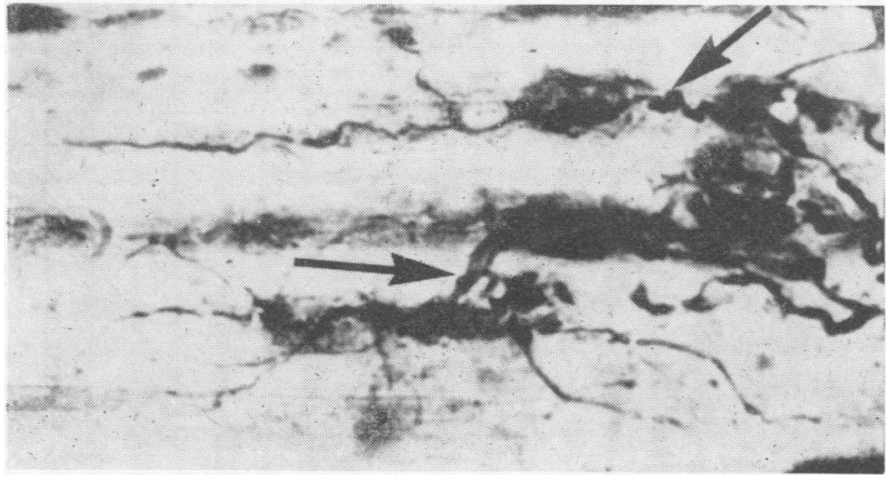

FIG. 6c

and end-plates. The points which are relevant here are that normally the end-plates are not easy to find, that the motor nerve terminals lie in the gutters of sarcolemmal membrane, and that subneural junctional folds project into a region of sarcoplasm rich in cell organelles (Doyère's hillock) close to the characteristic sole-plate nuclei. Nerve terminals normally always have subneural folds in their immediate vicinity. Cholinesterase can be demonstrated in the folds and in Schwann cell-axolemmal junctions.

In $\mathrm{m}$. biceps brachii of med mice aged 11 and 13 days which showed definite clinical disease the appearances of the motor end-plates were normal 


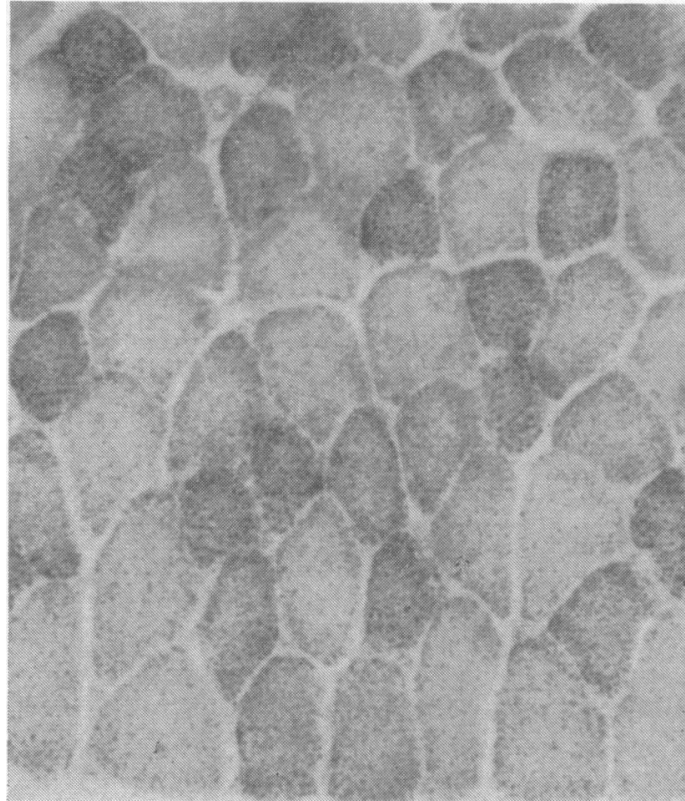

(a)

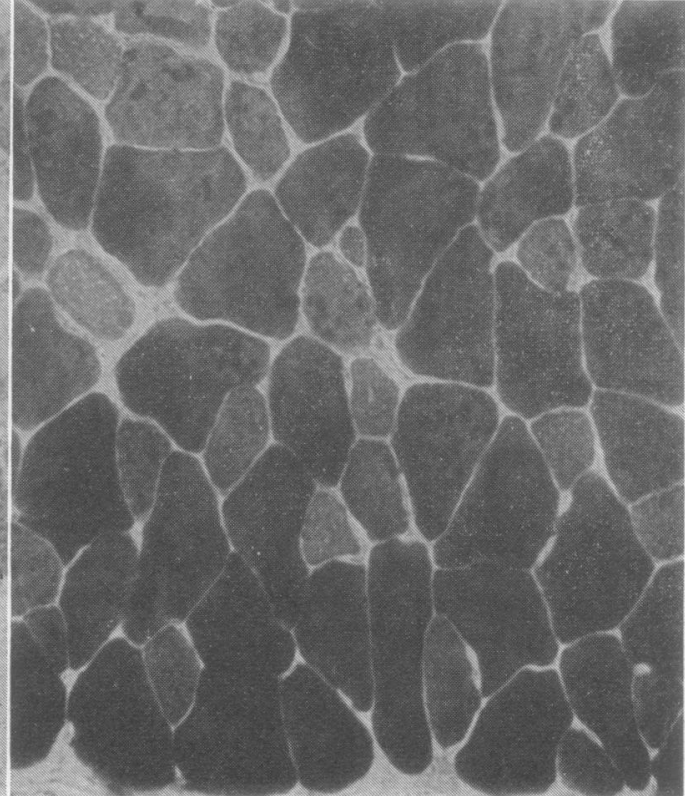

(b)

FIG. 7. Succinate dehydrogenase (a) and phosphorylase (b) reactions in the superficial part of $m$. biceps brachii of $\frac{7}{8}$ 을 normal mouse 19 days old. Most of the muscle fibres show a strong reaction for phosphorylase but few give a strong succinate dehydrogenase reaction. $\times 400$.

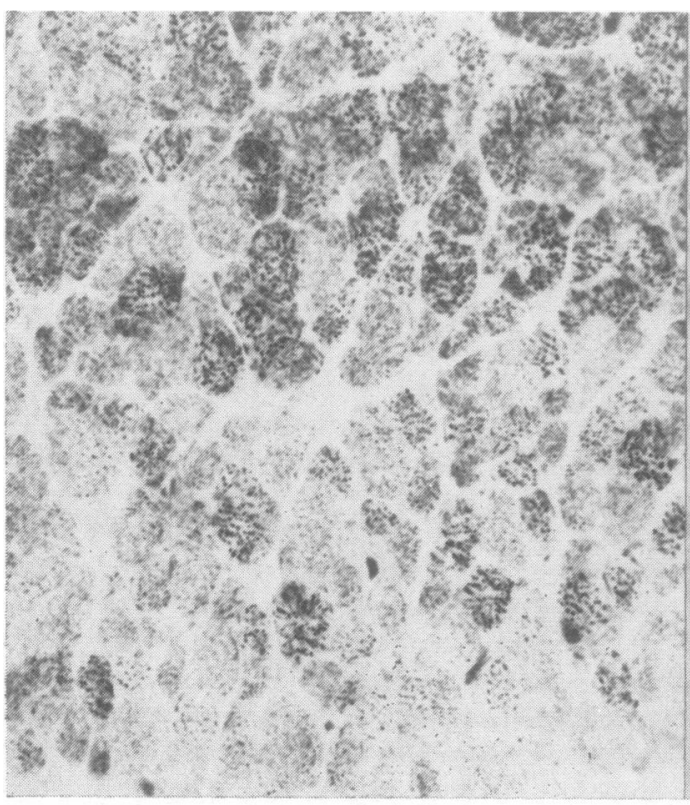

(a)

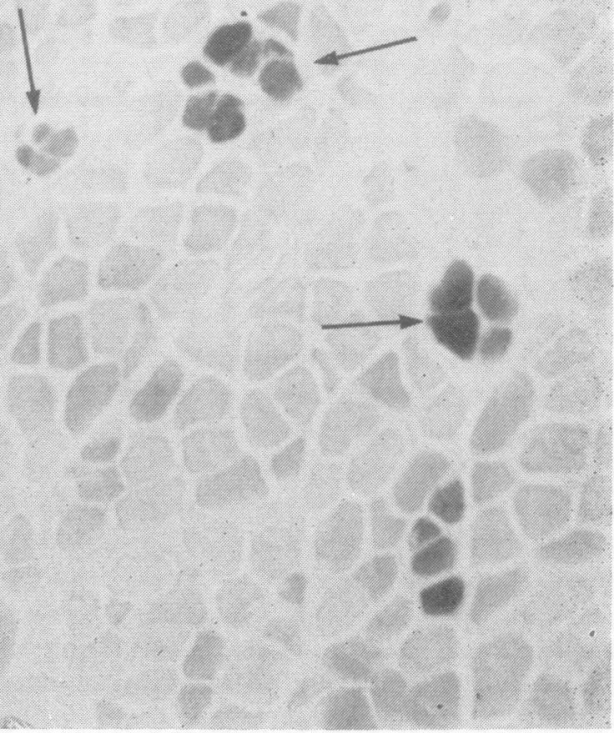

(b)

FIG. 8. M. biceps brachii of a 'med' mouse aged 19 days. Succinate dehydrogenase (a) is demonstrable in most muscle $\stackrel{N}{\mathrm{C}}$ fibres although they are atrophied. The phosphorylase reaction (b) is very weak except in intrafusal fibres of muscle $\vec{O}$ spindles (arrows). $\times 400$. 


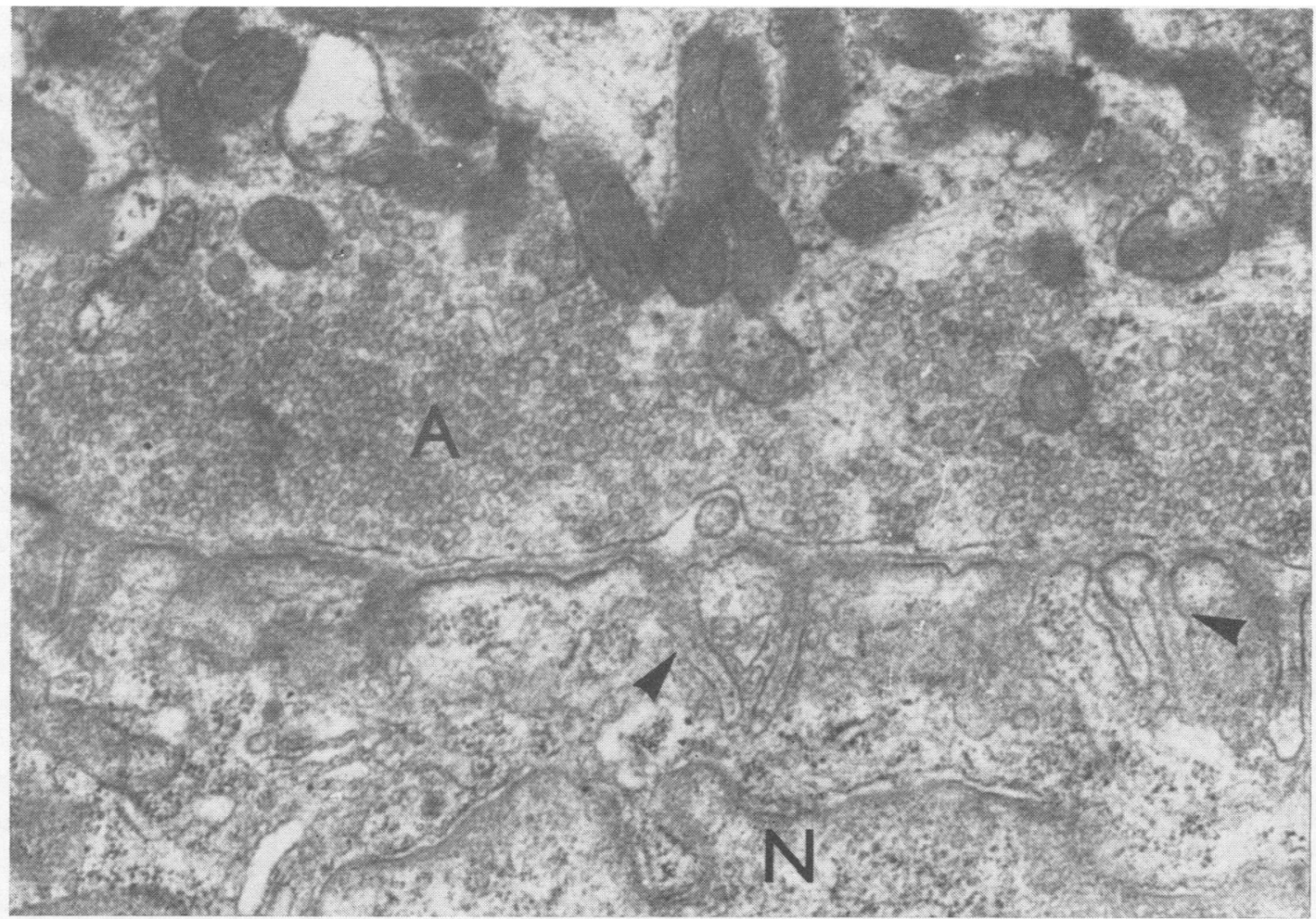

FIG. 9. Electron microscopic appearance of part of a motor end-plate in $m$. biceps brachii of a 'med' mouse aged 13 days. The appearance is like that of the normal. The terminal of the axon $(A)$ contains abundant mitochondria, neurofilaments and synaptic vesicles. The sarcolemmal membrane shows normal-looking subneural folds (arrows) and a sole-plate nucleus $(N)$ lies close to the synaptic membrane. $\times 35,000$.

(Fig. 9). The mice aged 18,21 , or 23 days showed abnormalities which were similar in all. Motor nerve terminals were more numerous than normal though their appearance was similar to the normal with abundant vesicles, filaments, and mitochondria. The relationship between many of the nerve terminals and the muscle fibres was abnormal. Many nerve terminals were situated in regions where sarcoplasmic organelles were not especially abundant and where no sole-plate nuclei were present-that is, they were outside the region of Doyère's hillock. In relation to some of these nerve terminals there wure abnormally few or shallow junctional folds of the sarcolemmal membrane (Fig. 10). It seemed likely that these were the terminals of the new nerve sprouts. The nerve sprouts were always closely enveloped by Schwann cells and their processes and cholinesterase reaction product was found in axolemmal-sarcolemmal junctions and in axolemmalSchwann cell junctions. Inhibitors of pseudocholin- esterase werenot used in electron microscopic studies. No denervated end-plates were seen in electron microscopic preparations. The appearance of axons and myelin sheaths of intramuscular nerve fibres was normal.

Within the muscle fibres the organization of the myofibrils was normal in the 11- and 13-day-old mice. Abnormalities which were found to develop in the muscle fibres were more conspicuous in the older ( 21 and 23 day) mice than at earlier stages of the disease. In some randomly scattered muscle fibres were dense bodies of varying size which were usually rounded and occasionally contained vesicles or membranes within them. The appearance of the dense bodies varied. They were either small and lying singly close to the $\mathrm{Z}$ line in the position where mitochondria are normally situated or in aggregates of larger dense masses which disorganized the internal structure of some muscle fibres (Fig. 11). The most severely atrophied muscle fibres usually 


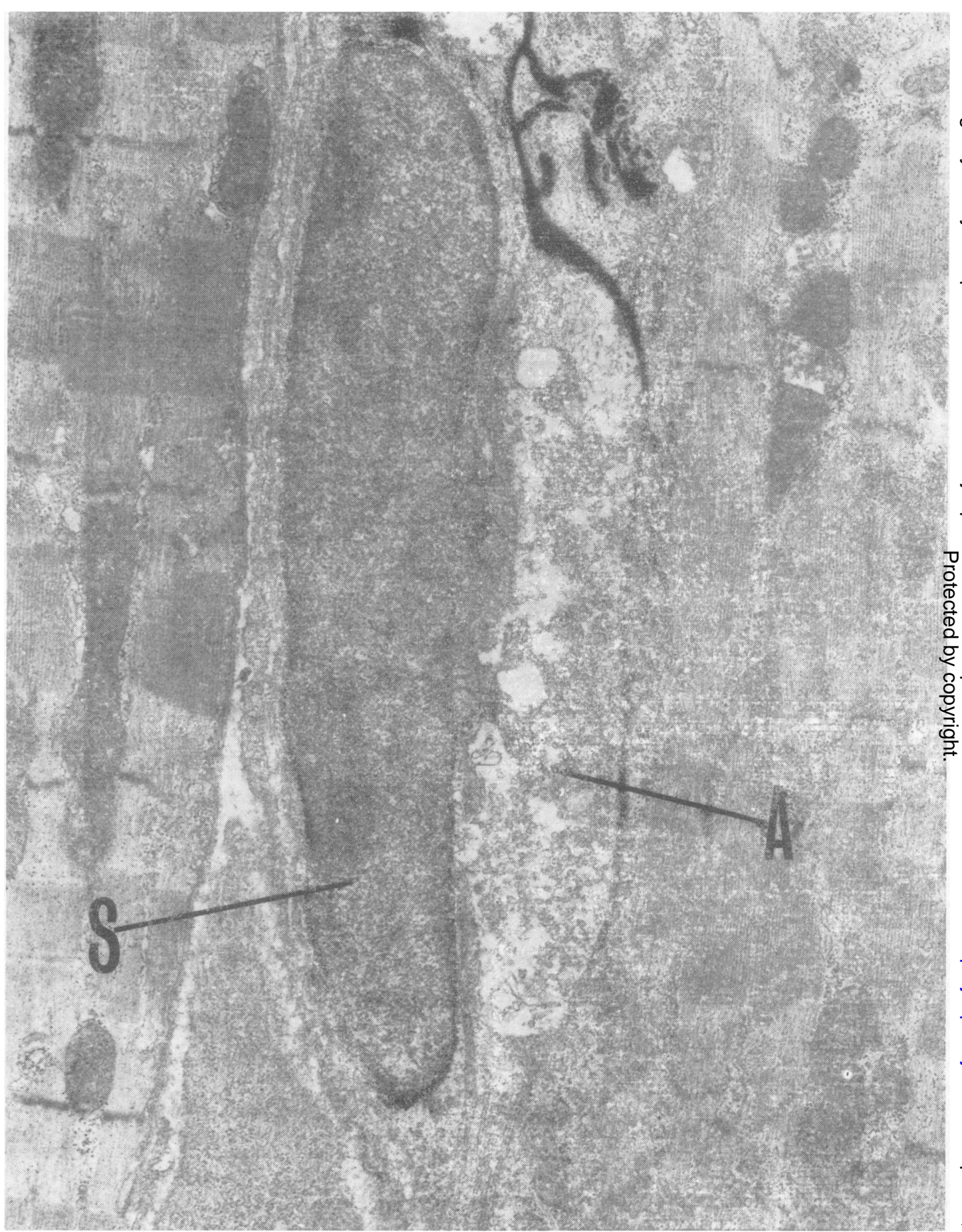

FIG. 10. Nerve terminal in biceps muscle of a ' $m$ ed' mouse 23 days old. Black precipitate from the cholinesterase reaction is concentrated mainly in the axolemmal-sarcolemmal junction. The axoplasm $(A)$ contains neurofilaments, mitochondria $\omega$ and vesicles. A Schwann cell $(S)$ and its processes lie over the nerve terminal. Subneural folds with cholinesterase reaction $\underset{O}{0}$ product are seen in part of the junctional area but in other parts folds are shallower than normal or entirely absent. The 0 sarcoplasm beneath the nerve terminal does not show a concentration of cell organelles. $\times 18,000$. 


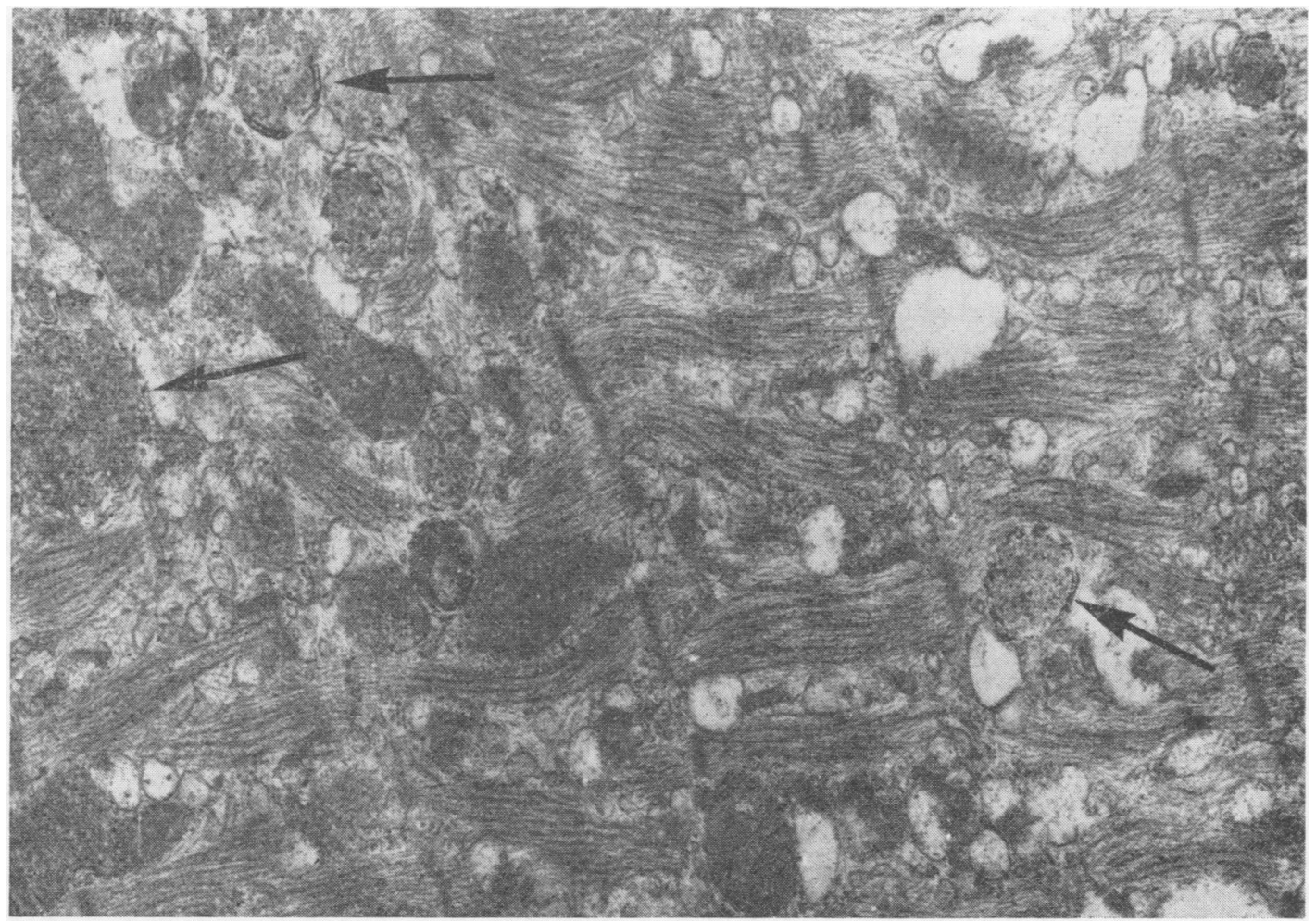

FIG. 11. Electron microscopic appearance of a muscle fibre of biceps brachii of a 'med' mouse 23 days old. Many dense bodies (arrows to some) are present. Some appear to be membrane-bound and contain vesicles, while others are aggregations of dense granules. The arrangement of the myofilaments is severely disorganized and dilatation of the sarcoplasmic reticulum is seen. $\times 27,000$.

contained the most aggregations of such dense bodies. Dilatation of the lateral cisternae of the triads was found in some muscle fibres. These dilated cisternae were few in number and scattered randomly in young med mice but were found in greater numbers in older mice. Lipid bodies were found in muscle fibres of both normal and affected mice but appeared to be more numerous in the latter.

\section{DISCUSSION}

The clinical signs of this hereditary disease are due to generalized muscular weakness. There are many other hereditary neurological diseases in mice (Sidman, Green, and Appel, 1965) from which the syndrome of the med mouse has to be distinguished. The progressive weakness leading to almost total flaccid paralysis, the absence of inco-ordination or other evidence of cerebellar ataxia, and the short survival time distinguish this disease from most of the mutants listed by Sidman et al. Progressive muscular weakness is also found in the wobbler mutant (Duchen, Falconer, and Strich, 1966; Duchen and Strich, 1968a) in which signs develop only at the fourth week of age and which may survive for many months. Muscle weakness is also characteristic of hereditary dystrophia muscularis (Michelson, Russell, and Harman, 1955) but the clinical signs are recognizable only at three to four weeks of age and survival is usually for many weeks (see also genetic appendix). However it is possible that a mutant similar to med does exist elsewhere and may have been named and allocated a gene symbol based only on the clinica! syndrome.

Histological examination of the central nervous system of med mice failed to show any morphological abnormalities, particularly of the ventral horn cells of the spinal cord, and no evidence of Wallerian degeneration was seen in ventral nerve roots or in peripheral nerves. The absence of such abnormalities in the lower motor neurone distinguished med from wobbler in which vacuolation and degeneration of motor nerve cells was an early change found in the brain-stem and spinal cord. In the skeletal muscles 
of med mice the main abnormality found in the muscle fibres themselves was atrophy. The atrophy of muscle fibres was progressive in that, with longer survival, fibres were smaller in the uniformly severely affected biceps, and in partially affected muscles such as triceps more fibres were atrophied than at earlier stages of the disease. There was no arrangement of atrophied fibres in groups or fascicles characteristic of partial motor denervation due to structural loss of motor nerve fibres as in human motor system diseases or peripheral neuropathies (Adams, Denny-Brown, and Pearson, 1962) and in the wobbler mouse. In none of the med mice examined histologically was necrosis of muscle fibres seen and the appearances were very unlike the changes which have been described in hereditary dystrophia muscularis in the mouse (Michelson et al., 1955; West and Murphy, 1960; Pearce and Walton, 1963; Banker, 1967) or in other primary myopathic diseases (Adams et al., 1962).

The changes in the innervation of skeletal muscle fibres of med mice consisted of sprouting from motor nerve terminals and the spread of demonstrable cholinesterase along the nerve sprouts. These changes are morphologically very like the terminal motor nerve sprouting which occurs during the prolonged period of muscle paralysis induced by the local injection of botulinum toxin in skeletal muscles of the mouse(Duchen and Strich, 1968b; Duchen, 1970). Botulinum toxin causes paralysis of skeletal muscle fibres without causing degeneration of preterminal or terminal nerve fibres. The similarity between the changes in muscles of the med mice and those occurring as a consequence of the local action of botulinum toxin suggested that the paralysis of muscle in the hereditary disease may be due to a functional interruption of neuromuscular transmission (Duchen and Strich, 1967; Duchen et al., 1967). In both conditions there develops an apparently paradoxical situation in which the paralysed and atrophied skeletal muscles contain an excess of motor nerve fibres. The electron microscopic observations have shown that the motor nerves and terminals of med mice contain a normallooking complement of vesicles and mitochondria. No denervated motor end-plates were seen. In the early stages of the disease the muscle fibres appeared normal and the abnormal multi-vesiculated bodies and dense bodies within muscle fibres in the late stages of the disease are best interpreted as being lysosomes and lipochrome granules developing during muscle fibre atrophy.

The principal enzyme histochemical change consisted of a progressive loss of phosphorylase activity, particularly in the superficial fibres of both biceps and triceps muscles where, in normal muscles, fibres show a strong phosphorylase reaction. Surgicall denervation of muscle in the mouse causes a similas reduction of phosphorylase activity in muscle fibres (author's observations).

The nerve sprouting in the muscles of med miced begins from the intact motor nerve terminals. Thers was no apparent sprouting from pretermina myelinated axons. In conditions in which it is knowi that motor nerve sprouting occurs after the structural loss of lower motor neurones (Edds, 1950; Hoffman $\overrightarrow{\vec{g}_{3}}$ 1950; Wohlfart and Hoffman, 1956; Duchen and Strich, 1968a) the pattern of innervation is unlike that seen in the present disease and in all these other. conditions the sprouting has been presumed to originate from surviving preterminal axons.

There is thus evidence that in hereditary motof end-plate disease there is a progressive loss of funce tional innervation of skeletal muscles which come to $\underline{\underline{\sigma}}$ contain an excess of motor nerve fibres and which show no positive evidence for a structural loss of motor axons. Positive evidence for structuraf denervation would have been degeneration of presw terminal axons, a fascicular distribution of atrophited muscle fibres, preterminal sprouting from surviving axons, or denervated motor end-plates on electpop microscopy. None of these was found. The patiog logical findings suggest that the disease is dueote progressive failure of neuromuscular transmissiap and progressive functional denervation of skelefa muscle. Recent electrophysiological studies (Duckefb and Stefani, in preparation) have shown that this in fact the case.

It is not known whether a human counterpart op hereditary motor end-plate disease exists. Possibly some cases of rapidly progressive or congenita hypotonia are due to a disorder of similar type as i this disease and are clinically indistinguishable fron? other forms of infantile hypotonia. In such case $\overrightarrow{\vec{s}}$ normal histological appearances of the spinal cor $\bar{\Phi}$ at necropsy should prompt study of the innervatio of skeletal musculature.

SUMMARY

Clinical and pathological studies (including light and electron microscopy) of 'hereditary motor ende plate disease' in the mouse are described. Thi disease is caused by a fully penetrant and fullg recessive autosomal gene med which is closely linked to the gene 'caracul' $\mathrm{Ca}$ in linkage group V Clinically it is characterized by progressive weakness. of skeletal muscles beginning at about eight to $10^{\top}$ days after birth and is fatal usually within 14 days్ల of onset. Light microscopic examination of the्E central nervous system failed to show any abnormo alities and the peripheral nerves were normat 
proximal to the motor end-plates. In early stages of the disease the structure of the motor nerve terminals and of skeletal muscle fibres appeared to be normal with both light and electron microscopy. With longer survival there was progressive atrophy of skeletal muscle fibres, particularly severe in proximal limb muscles. The histochemical reaction for phosphorylase became progressively weaker. Motor nerves innervating the atrophied muscle fibres showed marked terminal sprouting, the nerve sprouts growing beyond the normal confines of the motor end-plate. The sprouting nerve terminals contained abundant vesicles and mitochondria but the axolemmal-sarcolemmal junctions of these sprouts were deficient in subneural folds. The pathological findings suggest that this hereditary disease is due to progressive failure of neuromuscular transmission causing progressive functional denervation of skeletal muscle.

I should like to thank Mr. A. J. Davey, Mr. I. J. Stiff, and Mr. P. Marriott for the microscopic preparations and Mr. P. M. Taylor for help with photography. This work was supported by grants from the Research Fund of the Bethlem Royal and Maudsley Hospitals, the Muscular Dystrophy Associations of America, Inc., and the National Fund for Research into Crippling Diseases.

\section{ADDENDUM}

Since this paper was written the results of an investigation of med mice by Zacks, Sheff, Rhodes, and Saito (1969) have been published. These authors report that no abnormalities of the motor end-plate and no atrophy of muscle fibres were found. They conclude that the disease is primarily myopathic in nature. The most likely reason for the failure of Zacks et al. to observe significant pathological changes in the muscles of the med mouse may be that they did not observe that different muscles are affected with varying degrees of severity. Their studies were confined to intercostal and gastrocnemius muscles, which are not uniformly severely affected in this disease.

\section{REFERENCES}

Adams, R. D., Denny-Brown, D., and Pearson, C. M. (1962). Diseases of Muscle. 2nd ed., Henry Kimpton: London.
Banker, B. Q. (1967). A phase and electron microscopic study of dystrophic muscle. I. The pathological changes in the twoweek-old Bar Harbor 129 dystrophic mouse. J. Neuropath. exp. Neurol., 26, 259-275.

Beesley, R. A., and Daniel, P. M. (1956). A simple method for preparing serial blocks of tissue. J. clin. Path., 9, 267-268.

Duchen, L. W. (1970). Changes in motor innervation and cholinesterase localization induced by botulinum toxin in skeletal muscle of the mouse: differences between fast and slow muscles. J. Neurol. Neurosurg. Psychiat., 33, 40-54.

- , Falconer, D. S., and Strich, S. J. (1966). Hereditary progressive neurogenic muscular atrophy in the mouse. J. Physiol. (Lond.), $183,53-55 P$.

Searle, A. G., and Strich, S. J. (1967). An hereditary motor endplate disease in the mouse. J. Physiol. (Lond.), 189, 4-6P.

- , and Strich, S. J. (1967). Changes in the pattern of motor innervation of skeletal muscle in the mouse after local injection of Clostridium botulinum toxin. J. Physiol. (Lond.), 189, 2-4P.

- (1968a). An hereditary motor neurone disease with progressive denervation of muscle in the mouse: the mutant 'wobbler'. J. Neurol. Neurosurg. Psychiat., 31, 535-542.

- 1 - (1968b). The effects of botulinum toxin on the pattern of innervation of skeletal muscle in the mouse. Quart. J. exp. Physiol., 53, 84-89.

Edds, M. V. Jr. (1950). Collateral regeneration of residual motor axons in partially denervated muscles. J. exp. Zool., 113, 517551.

Henderson, J. R. (1967). The use of silver for intensifying sulfide deposits in the cholinesterase technique. Stain Tech., 42, 101102.

Hoffman, H. (1950). Local re-innervation in partially denervated muscle: a histo-physiological study. Aust.J. exp. Biol. med. Sci., 28, 383-397.

Koelle, G. B., and Friedenwald, J. S. (1949). A histochemical method for localizing cholinesterase activity. Proc. Soc. exp. Biol. Med., 70, 617-622.

Lewis, P. R. (1961). The effect of varying the conditions in the Koelie technique. Bibl. anat. (Basel), 2, 11-20.

Michelson, A., Russell, E. S., and Harman, P. J. (1955). Dystrophia muscularis: a hereditary primary myopathy in the house mouse. Proc. nat. Acad. Sci. (Wash.), 41, 1079-1084.

Namba, T., Nakamura, T., and Grob, D. (1967). Staining for nerve fiber and cholinesterase activity in fresh frozen sections. Amer. J. clin. Path., 47, 74-77.

Pearce, G. W., and Walton, J. N. (1963). A histological study of muscle from the Bar Harbor strain of dystrophic mice. J. Path. Bact., 86, 25-33.

Pearse, A. G. E. (1960). Histochemistry. Theoretical and Applied. 2nd ed., Churchill: London.

Schofield, G. C. (1960). Experimental studies on the innervation of the mucous membrane of the gut. Brain, 83, 490-514.

Searle, A. G. (1962). Mouse News Lett., 27, 34

Sidman, R. L., Green, M. C., and Appel, S. H. (1965). Catalog of the Neurological Mutants of the Mouse, Harvard Univ. Press Cambridge, Mass.

West, W. T., and Murphy, E. D. (1960). Histopathology of hereditary progressive muscular dystrophy in inbred strain 129 mice. Anat. Rec., 137, 279-295.

Wohlfart, G., and Hoffman, H. (1956). Reinnervation of muscle fibers in partially denervated muscles in Theiler's encephalomyelitis of mice (mouse poliomyelitis). Acta psychiat. scand., 31, 345-365.

Zacks, S. I., Sheff, M. F., Rhodes, M., and Saito, A. (1969). MED myopathy. A new hereditary myopathy of mice. Lab. Invest., 21, 143-153.
The med gene arose by spontaneous mutation in a stock homozygous for non-agouti $(a)$, brown $(b)$, chinchilla $\left(c^{c h}\right)$, pink-eyed dilute $(p)$, dilute $(d)$, short-ear (se), and piebald $(s)$. A technician, Miss Daphne Buck, noticed that a mouse in litter CT/478.1 born 23 October 1958, was making wriggling seal- 